\title{
PET com Correção de Atenuação
}

Os métodos de correção de atenuação anteriormente descritos podem ser aplicados tanto às técnicas tomográficas utilizando emissão de fóton único (SPECT) como às técnicas por emissão de pósitrons (PET). No entanto, no caso da correção de atenuação por método de transmissão (medida) a fonte externa utilizada vai variar dependendo da técnica que se quer corrigir. No caso da $\mathrm{PET}$, as fontes que podem ser utilizadas são o césio-137 $\left({ }^{137} \mathrm{Cs}\right)$, o germânio-68 $\left({ }^{68} \mathrm{Ge}\right)$ e os raios- $X$, quando disponíveis. É importante que a energia dos fótons emitidos pelas fontes utilizadas na transmissão seja próxima àquelas dos fótons emitidos pelo radioisótopo injetado, $511 \mathrm{keV}$ no caso dos emissores de pósitrons e $140 \mathrm{keV}$ no caso do tecnécio-99m. 0 césio-137 emite fóton com energia de $662 \mathrm{keV}$, e, apesar de esta energia não ser idêntica àquela emitida na degradação dos pósitrons (511 keV), existem maneiras para compensar esta diferença. A grande vantagem da utilização do césio-137 é sua meiavida física longa, não requerendo trocas da fonte durante a vida útil do equipamento. 0 germânio-68, também um emissor de pósitrons, emite radiação gama de $511 \mathrm{keV}$.

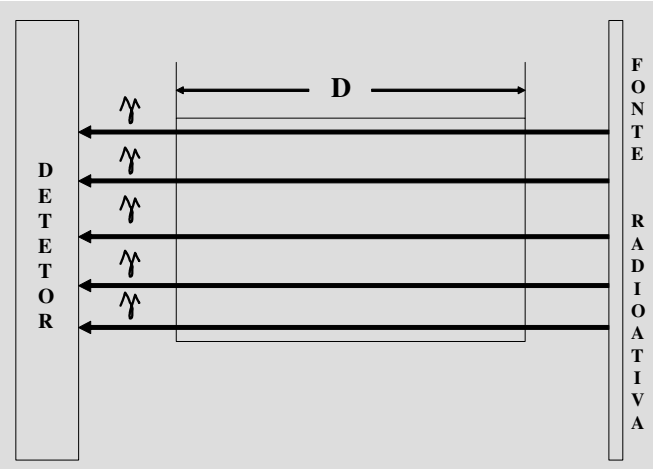

Figura 4a - Modelo esquemático mostrando que a atenuação medida estima a atenuação em uma determinada direção, considerando que o fóton irá percorrer toda a extensão do paciente.

A grande desvantagem da utilização do germânio-68 é sua meia-vida física de 288 dias, que requer que a fonte seja trocada periodicamente. A utilização de raios- $X$ para realizar a correção de atenuação para PET é mais recente, e uma das dificuldades atuais no uso desta técnica é extrapolar os mapas de correção de atenuação obtidos com a baixa energia emitida pelas ampolas (ao redor de $140 \mathrm{keV}$ ) para aqueles que seriam obtidos utilizando-se radiação de $511 \mathrm{keV} \mathrm{1,2}$, além da aquisição emissão/ transmissão ser seqüencial, o que pode acarretar artefatos por desalinhamento das informações.

Além da maior energia da radiação gama emitida, a PET possui algumas outras diferenças em relação à medicina nuclear convencional que facilitam com que se corrijam as alterações decorrentes de atenuação.

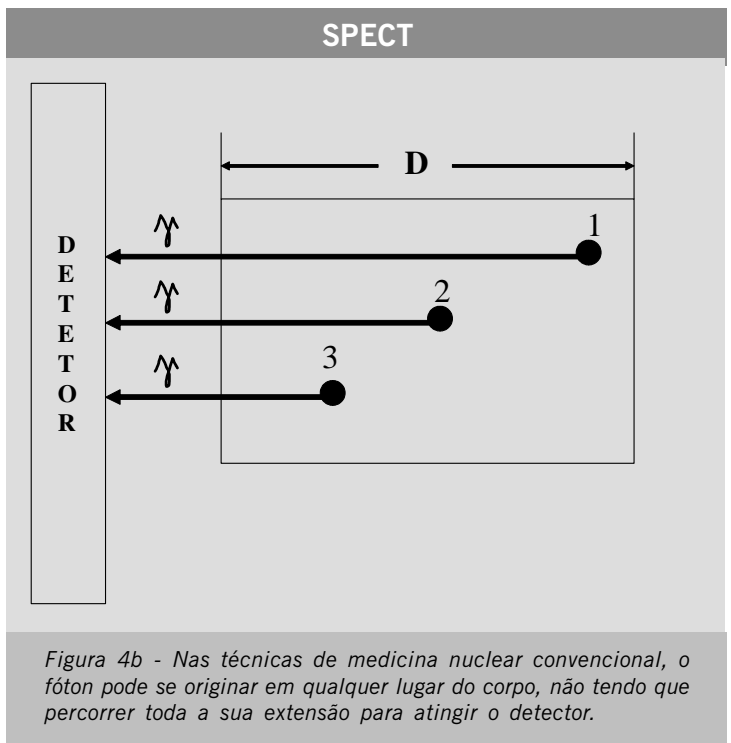

Nas técnicas de medicina nuclear convencional, é impossível saber a real distância percorrida por um fóton antes de atingir o sistema de detecção, dificultando a utilização dos métodos de correção de atenuação, uma vez que esta variável é fundamental para o fenômeno de atenuação. Na técnica por emissão de pósitrons, como a metodologia se baseia na detecção em coincidência dos dois fótons gama que são emitidos em sentidos opostos, ela funciona como se um evento radioativo percorresse toda a extensão do paciente antes de atingir o detector, o que facilita a utilização de métodos de correção (figura 4).

No caso específico da avaliação de viabilidade miocárdica utilizando a PET, a correção de atenuação

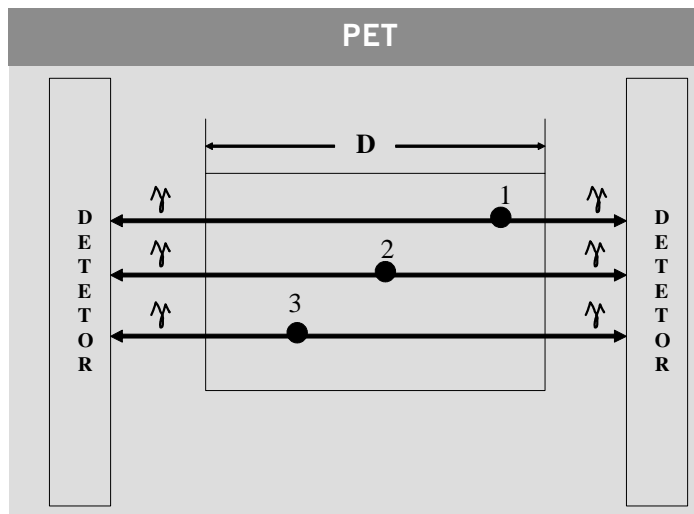

Figura 4c - Desta maneira, os mapas de atenuação criados pelo sistema de transmissão não representam, de maneira adequada, o que realmente acontece com o fóton emitido pelo paciente, gerando um erro do método. Na técnica de tomografia por emissão de pósitrons, como são emitidos dois fótons em sentidos opostos, . antes de atingir os detectores, o que torna os mapas de transmissão mais adequados para esta última técnica. 
tem-se mostrado útil na interpretação das imagens, sendo que a ausência de correção poderá levar a interpretações equivocadas do exame (figura 4). É fundamental lembrar que, quando utilizamos a glicose radioativa para a avaliação da viabilidade do miocárdio, as imagens podem ser adquiridas com equipamentos PET dedicados, com câmaras de cintilação com circuito de coincidência (equipamentos híbridos PET-SPECT) e utilizando colimadores de ultra-alta energia ${ }^{3-5}$. Quando se utilizam os colimadores de ultra-alta energia, não se emprega mais a detecção em coincidência para posicionar espacialmente o evento radioativo, mas, sim, o colimador funcionando como uma técnica de medicina nuclear convencional. Nesta última situação, não se realiza a correção de atenuação medida e não existem mecanismos desenvolvidos para tal fim. No entanto, estudos mostraram que a atenuação neste tipo de sistema é menos problemática, sendo que esta forma de aquisição de imagem se mostrou superior aos sistemas híbridos (PET-SPECT), mesmo com correção de atenuação, para a avaliação da viabilidade miocárdica. Mas os resultados obtidos com a utilização dos colimadores são inferiores àqueles obtidos utilizando-se os equipamentos dedicados à realização de imagens $\mathrm{PET}^{4,6}$.

As técnicas de correção de atenuação têm-se mostrado úteis na avaliação das imagens de medicina nuclear em cardiologia, tanto no que se refere às técnicas de SPECT como no que se refere a PET. Na pesquisa de viabilidade miocárdica com FDG- ${ }^{18} \mathrm{~F}$ (figura 5), a utilização de técnicas de correção de atenuação é um procedimento importante, principalmente quando se utilizam os sistemas híbridos (PET-SPECT).

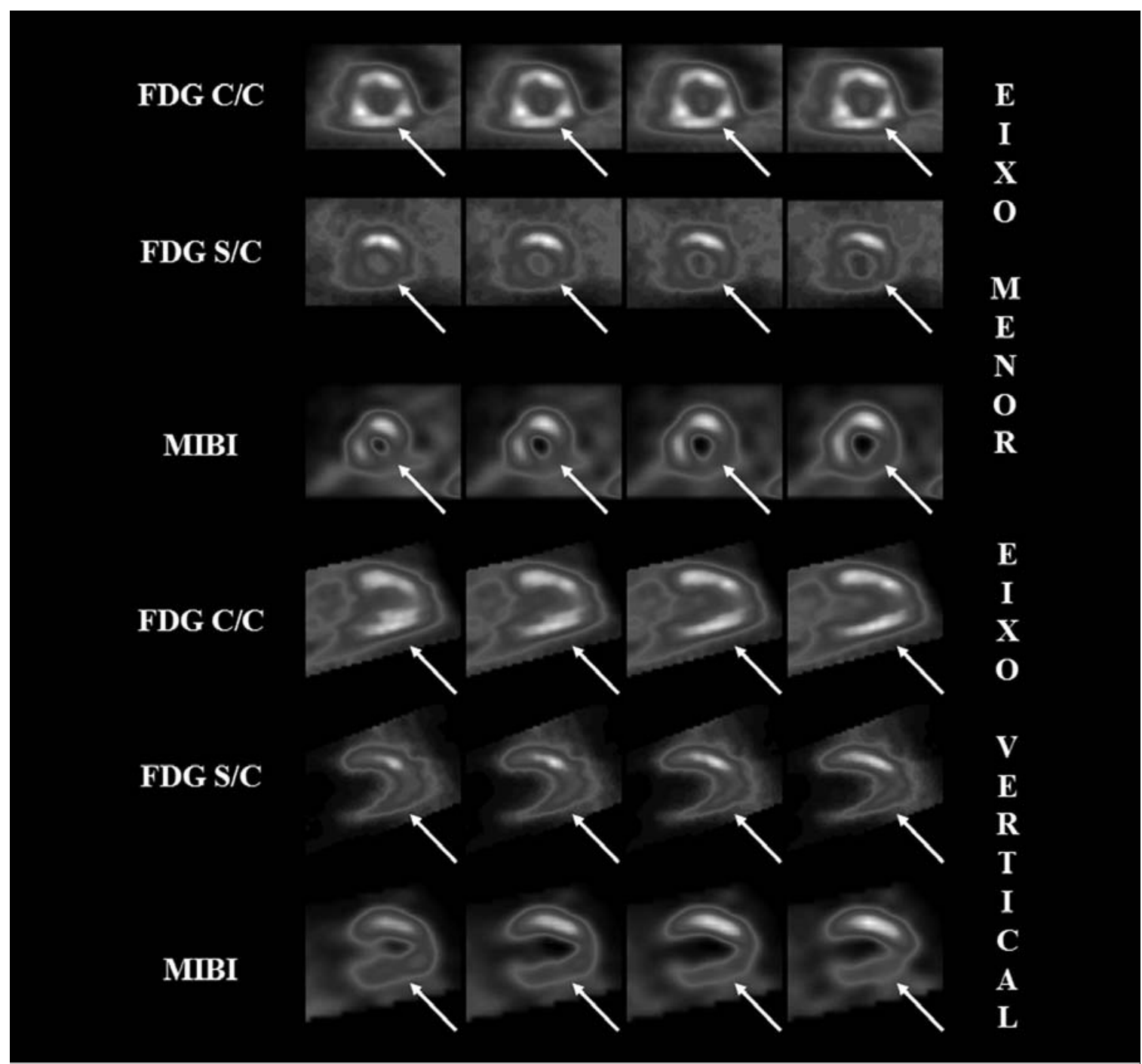

Figura 5 - Exemplo de como a utilização de correção de atenuação pode melhorar a acurácia do exame. Observar o eixo menor e o eixo maior vertical de exame de FDG- ${ }^{18}$ FPET realizado com correção de atenuação (C/C) e sem correção de atenuação (S/C) e do exame de perfusão do miocárdio realizado com sestamibi-99m Tc. Notamos que o exame com correção de atenuação mostra metabolismo na parede ínfero-dorsal (seta), o qual não é observado na imagem sem correção de atenuação. 


\section{REFERÊNCIAS}

1. Burger C, Goerres G, Schoenes S, Buck A, Lonn AH, Von Schulthess GK. PET attenuation coefficients from CT images: experimental evaluation of the transformation of CT into PET 511-keV attenuation coefficients. Eur J Nucl Med Mol Imaging 2002; 29: 922-27.

2. Kamel E, Hany TF, Burger $\mathrm{C}$ et al. CT vs $68 \mathrm{Ge}$ attenuation correction in a combined PET/CT system: evaluation of the effect of lowering the CT tube current. Eur J Nucl Med Mol Imaging 2002; 29: 346-50.

3. Bax JJ, Patton JA, Poldermans D, Elhendy A, Sandler MP. 18 Fluorodeoxyglucose imaging with positron emission tomography and single photon emission computed tomography: cardiac applications. Semin Nucl Med 2000; 30: 281-98.
4. Mabuchi M, Kubo N, Morita Ket al. Value and limitation of myocardial fluorodeoxyglucose single photon emission computed tomography using ultra-high energy collimators for assessing myocardial viability. Nucl Med Commun 2002; 23: 879-85.

5. Nowak B, Zimny M, Schwarz ER et al. Diagnosis of myocardial viability by dual-head coincidence gamma camera fluorine-18 fluorodeoxyglucose positron emission tomography with and without non-uniform attenuation correction. Eur J Nucl Med 2000; 27:1501-08.

6. Hasegawa S, Uehara T, Yamaguchi $\mathrm{H}$ et al. Validity of $18 \mathrm{~F}$ fluorodeoxyglucose imaging with a dual-head coincidence gamma camera for detection of myocardial viability. J Nucl Med 1999; 40:1884-92. 